\title{
Late (5-20 years) outcomes after STA-MCA anastomosis and encephalo-duro-myo-arterio-pericranial synangiosis in patients with moyamoya disease
}

\author{
Satoshi Kuroda, MD, PhD, ${ }^{1,2}$ Naoki Nakayama, MD, PhD, ${ }^{2}$ Shusuke Yamamoto, MD, ${ }^{1}$ \\ Daina Kashiwazaki, MD, PhD, ${ }^{1}$ Haruto Uchino, MD, PhD, ${ }^{1,2}$ Hisayasu Saito, MD, PhD, ${ }^{1,2}$ \\ Emiko Hori, MD, PhD, ${ }^{1}$ Naoki Akioka, MD, ${ }^{1}$ Naoya Kuwayama, MD, PhD, ${ }^{1}$ and \\ Kiyohiro Houkin, MD, PhD²
}

'Department of Neurosurgery, Graduate School of Medicine and Pharmaceutical Science, University of Toyama, Toyama; and 2Department of Neurosurgery, Hokkaido University Graduate School of Medicine, Sapporo, Japan

OBJECTIVE Surgical revascularization is known to reduce the incidence of further ischemic and hemorrhagic events in patients with moyamoya disease, but the majority of previous studies report only short-term $(<5$ years) outcomes. Therefore, in this study the authors aimed to evaluate late (5-20 years) outcomes of moyamoya patients after superficial temporal artery to middle cerebral artery (STA-MCA) anastomosis and indirect bypass (encephalo-duro-myo-arteriopericranial synangiosis [EDMAPS]).

METHODS Cumulative incidences of late morbidity/mortality and disease progression were evaluated among 93 patients who underwent STA-MCA anastomosis and EDMAPS. All of the patients were prospectively followed up for longer than 5 years postsurgery ( $10.5 \pm 4.4$ years). There were 35 pediatric and 58 adult patients. Initial presentation included transient ischemic attack/ischemic stroke in 80 patients and hemorrhagic stroke in 10 patients, and 3 patients were asymptomatic. Surgery was performed in a total of 141 hemispheres. Follow-up MRI/MRA was performed within a 6 - or 12-month interval during the follow-up periods.

RESULTS During the follow-up periods, 92/93 patients were free from any stroke or death, but 1 patient had a recurrence of hemorrhagic stroke $(0.10 \%$ per patient-year). Disease progression occurred in the territory of the contralateral carotid or posterior cerebral artery (PCA) in 19 hemispheres of 15 patients (1.5\% per patient-year). The interval between initial surgery and disease progression varied widely, from 0.5 to 15 years. Repeat bypass surgery for the anterior and posterior circulation resolved ischemic attacks in all 10 patients.

CONCLUSIONS The study results indicate that STA-MCA anastomosis and EDMAPS would be the best choice to prevent further ischemic and hemorrhagic stroke for longer than 10 years on the basis of the demonstrated widespread improvement in cerebral hemodynamics in both the MCA and ACA territories in the study patients. However, after 10 years postsurgery regular follow-up is essential to detect disease progression in the territory of the contralateral carotid artery and PCA and prevent late cerebrovascular events.

https://thejns.org/doi/abs/10.3171/2019.12.JNS192938

KEYWORDS moyamoya disease; late outcome; combined bypass; disease progression; vascular disorders

$\mathrm{M}$ OYAMOYA disease often causes ischemic and/or hemorrhagic events in both children and adults. Radiologically, the terminal portion of the internal carotid artery (ICA) and its main branches within the circle of Willis show progressive occlusion associated with the formation of a fine vascular network (moyamoya vessels) at the base of the brain..$^{20,35}$ Most pediatric moyamoya patients suffer transient ischemic attack (TIA) and/ or ischemic stroke due to persistent cerebral ischemia in the territory of the involved arteries. On the other hand, a

ABBREVIATIONS CBF = cerebral blood flow; CVR = cerebral blood reactivity; EDAS = encephalo-duro-arterio-synangiosis; EDMAPS = encephalo-duro-myo-arteriopericranial synangiosis; EDPS = encephalo-duro-pericranio-synangiosis; EMS = encephalo-myo-synangiosis; ICA = internal carotid artery; MCA = middle cerebral artery; $\mathrm{mRS}=$ modified Rankin Scale; PCA = posterior cerebral artery; STA = superficial temporal artery; TIA = transient ischemic attack.

SUBMITTED October 29, 2019. ACCEPTED December 17, 2019.

INCLUDE WHEN CITING Published online March 13, 2020; DOI: 10.3171/2019.12.JNS192938. 
significant number of adult patients are known to develop hemorrhagic stroke. ${ }^{20}$ It is widely recognized that surgical revascularization improves cerebral blood flow (CBF) and metabolism and reduces the incidence of further ischemic and hemorrhagic events. ${ }^{16,19,22,28,29}$ Previously, three types of surgical procedures have been reported for moyamoya disease: direct, indirect, and combined bypass. Direct bypass procedures such as superficial temporal artery to middle cerebral artery (STA-MCA) anastomosis are known to quickly improve $\mathrm{CBF}$ and reduce ischemic attacks after surgery. Indirect bypass induces spontaneous angiogenesis between the brain surface and vascularized donor tissues such as the dura matter and temporal muscle. Combined bypass includes both procedures. ${ }^{19,20}$ However, each surgical procedure is known to improve patient outcomes. For example, indirect bypass increases the incidence of perioperative ischemic events because of the lack of surgical collaterals just after surgery, and direct or combined bypass improves cerebral hemodynamics just after surgery. ${ }^{13}$ Furthermore, surgery through a small craniotomy centered on the temporoparietal region is closely related to poor intellectual outcome in pediatric moyamoya disease patients because of persistent cerebral ischemia in the frontal lobe, even after surgery. ${ }^{21}$ Furthermore, a majority of surgical procedures are targeted to the MCA territory, but it is also important to revascularize the ACA territory in order to resolve sudden spontaneous falls, known as drop attacks, and preserve intellectual function in both pediatric and adult patients with moyamoya disease. ${ }^{11,22}$ For these purposes, some reports have recommended STAACA anastomosis to improve hemodynamic compromise in the ACA territory, but this procedure is still challenging because of the small caliber and fragility of the recipient vessels. Dissection of a longer graft of the STA may also cause delayed wound healing or scalp necrosis..$^{14}$

To further improve long-term outcomes in moyamoya patients, we developed a novel indirect bypass procedure, encephalo-duro-myo-arterio-pericranial synangiosis (EDMAPS). In this procedure, the frontal pericranium is used as donor tissue to widely cover the frontal lobe and improve cerebral hemodynamics in both the MCA and ACA territories. As reported previously, we performed STA-MCA anastomosis and EDMAPS in a total of 123 hemispheres of 75 patients ( 28 children and 47 adults). ${ }^{22}$ The incidences of mortality and morbidity were $0 \%$ and $5.7 \%$, respectively. Follow-up blood flow studies clearly showed that the procedures were effective in improving $\mathrm{CBF}$ and reactivity to acetazolamide in both the MCA and ACA territories. During a mean period of 67 months, the annual risk of cerebrovascular events during the follow-up periods was $0 \%$ in pediatric patients. Only 1 adult patient developed a cerebral infarction in the occipital lobe because of disease progression in the PCA (annual risk, $0.4 \%$ per year). Clinical results after STA-MCA anastomosis and EDMAPS were considered better than those after previously reported procedures. . $^{2,5,6,10,27,32}$ Therefore, we reported EDMAPS to be an "ultimate" indirect bypass. ${ }^{22}$

However, most previous studies have evaluated only short-term $(<5$ years) outcomes after surgery, including our previous study. As a result, before we undertook this investigation the impact of the EDMAPS indirect bypass procedure on long-term ( $>5$ years) outcomes was unknown. We believe that therapeutic benefits of surgical treatment of moyamoya should be assessed for a much longer time, because a majority of surgically treated patients are children or young adults. In addition, few studies have evaluated disease progression in the nonoperated hemispheres and the posterior cerebral artery (PCA) for an extended postsurgical time period. Therefore, in this study we aimed to evaluate the long-term (5-20 years) clinical and radiological outcomes after STA-MCA anastomosis and EDMAPS for moyamoya patients.

\section{Methods \\ Patients}

This study was approved by the local institutional review board, and informed consent was obtained from all participants or their legal representatives. This study included a total of 105 patients who underwent STAMCA anastomosis and EDMAPS for moyamoya disease at Toyama University Hospital or Hokkaido University Hospital between April 1998 and August 2014. All patients fulfilled the radiological criteria for the diagnosis of moyamoya disease set by the Research Committee on Moyamoya Disease established by the Ministry of Health, Labor and Welfare of Japan. ${ }^{34}$ Patients with secondary moyamoya syndrome associated with underlying disorders such as trisomy 21 and neurofibromatosis type 1 were excluded from this study.

Surgical revascularization was performed in 157 hemispheres of the 105 patients. Of these, 4 patients suffered major ischemic or hemorrhagic stroke within 30 days after surgery (3.8\% per patient or $2.5 \%$ per surgery) and were excluded from the study. Another 8 patients (7.6\%) were lost during follow-up within 5 years after surgery mainly because they moved to a new address. The remaining 93 patients $(88.6 \%)$ were prospectively followed up for 5 years or longer after surgery and their outcomes were precisely analyzed in this study.

\section{Preoperative Examinations}

The following examinations were performed in all patients: plain CT, MRI, MRA, cerebral angiography, and SPECT/PET. Using a 1.5- or 3.0-Tesla MR apparatus, multisequence MRI and 3D time-of-flight (3D-TOF) MRA were performed, including T1-, T2-, T2*-, and diffusion-weighted images and FLAIR imaging. Using ${ }^{123} \mathrm{I}-$ iodoamphetamine SPECT or ${ }^{15} \mathrm{O}$-gas PET, CBF and cerebral blood reactivity (CVR) were quantitatively measured before and after intravenous injection of acetazolamide. ${ }^{23}$

\section{Surgical Indication}

In ischemic-type moyamoya disease, reduced CVR in response to acetazolamide was considered a key finding to identify a critical decrease of cerebral perfusion pressure in the involved hemispheres. ${ }^{22}$ In hemorrhagic-type moyamoya disease, surgical revascularization was indicated in both hemispheres on the basis of the criteria used in the Japan Adult Moyamoya (JAM) Trial. ${ }^{30}$ Surgical revascularization was indicated for asymptomatic patients when their angiographical stage was markedly advanced in as- 
sociation with dense cerebral ischemia or silent microbleeds increased in number during the follow-up period. ${ }^{18}$

\section{Perioperative Management}

All patients received an extracellular fluid intravenous drip (500-1000 ml) overnight prior to surgery to prevent dehydration-induced ischemic stroke during and after surgery. During and following the induction of general anesthesia, systolic blood pressure was kept between 100 and $120 \mathrm{~mm} \mathrm{Hg}$, and $\mathrm{PaCO}_{2}$ was strictly maintained around $40 \mathrm{~mm} \mathrm{Hg} .^{22}$

Using SPECT, we qualitatively measured CBF immediately after surgery and 2 days and 7 days postsurgery to promptly detect postoperative hyperperfusion. Blood pressure was controlled at a level up to $20 \%$ lower than usual when the operated hemisphere was judged as having hyperperfusion..$^{38}$

\section{Surgical Procedures}

STA-MCA anastomosis and EDMAPS were performed as described previously, with minor modifications. ${ }^{22}$ Following the induction of general anesthesia, the patients were placed in the supine position with the head fixed with a 3-point fixation device. The course of the STA was detected using a Doppler ultrasound probe. The hair was partially shaved, and a skin incision was made along the course of the main trunk and parietal branch of the STA. These were dissected from the surrounding tissues under a surgical microscope and were kept patent. Then, the scalp flap was reflected laterally, and the frontal branch of the STA was also carefully dissected. The galeal tissue over the frontal branch of the STA was repaired with absorbable surgical sutures to prevent delay of wound healing.

The vascularized frontal pericranium was then prepared for subsequent encephalo-pericranio-synangiosis (EPS) using the cranium periosteum and the overlying loose areolar layer. Then, a vascularized flap of temporal muscle was prepared for encephalo-myo-synangiosis (EMS). A standard frontotemporal craniotomy extending into the frontal area was performed, preserving the middle meningeal artery (MMA). ${ }^{9}$ The dura was incised and rolled back by preserving the main branches of the MMA.

An STA-MCA single or double end-to-side anastomosis was then performed using 10-0 or 11-0 nylon threads on the brain surface. The authors preferred 11-0 nylon threads for pediatric patients younger than 10 years. Procedures were performed very carefully, which was important to create a successful STA-MCA anastomosis because the diameters of the recipient vessels were very small $(0.4-1.0 \mathrm{~mm})$ and their walls were very thin. Blue dye was put onto the surface of the cut ends of the donor and recipient vessels to enable their clear visualization. ${ }^{15}$ A green silicone sheet was inserted beneath the recipient vessel for the same purpose. Usually, 12 to 16 interrupted sutures were made to complete the STA-MCA anastomosis, and 20-30 minutes was required for recipient vessel clamping. ICG (indocyanine green) videoangiography was performed to confirm the patency of the STA-MCA anastomosis during surgery.

Encephalo-duro-synangiosis (EDS) was performed by turning the dural flaps inward toward the epi-arachnoid space to facilitate the development of surgical collaterals between the outer surface of the dura and the brain. Then, the dural window was covered with both the temporal muscle and pericranial flap. Cranioplasty was performed as usual, using titanium plates, but absorbable fixation plates were employed for pediatric patients younger than 10 years to avoid disturbing the normal growth of the skull. The wound was closed in a layer-by-layer fashion. The total operation time ranged from 5 to 6 hours.

\section{Follow-Up}

All patients were prospectively checked at an outpatient clinic for a mean of $10.5 \pm 4.4$ years (range 5-20 years). MRI and MRA were repeated every 6 or 12 months. In this study, the primary endpoint was any ischemic or hemorrhagic stroke during the follow-up period. TIA with fresh cerebral infarction on diffusion-weighted MRI was considered an ischemic stroke even if clinically transient neurological deficits resolved within 24 hours after the onset. The secondary endpoint was disease progression during the follow-up periods.

\section{Results}

In this study we aimed to evaluate late (longer than 5 years) outcome in 93 moyamoya patients who underwent STA-MCA anastomosis and EDMAPS. Of these 93 patients, 35 were pediatric (age $<18$ years) and 58 were adult patients. There were 29 male and 65 female patients. The mean \pm SD age for all patients was $30.0 \pm 19.0$ years (range 1-70 years). The clinical diagnosis included TIA or ischemic stroke in 80 patients, hemorrhagic stroke in 10, and asymptomatic in 3 patients. Precise data are presented in Table 1.

\section{Clinical Outcome}

STA-MCA anastomosis and EDMAPS were performed on 141 hemispheres of 93 patients. There was no mortality. During perioperative periods, a total of 19 complications occurred (13.5\%), including ischemic stroke $(n=5)$, symptomatic hyperperfusion $(n=6)$, transient cheiro-oral syndrome $(n=5)$, and delay of wound healing $(n=3)$. Of 5 cases of ischemic stroke, 1 case occurred before surgery in a 1-year-old patient. Ischemic stroke-related neurological deficits were mild but persisted in 3 of 5 cases for longer than 30 days. Symptomatic hyperperfusion resolved within 7 days after surgery in all 6 cases. Transient cheirooral syndrome disappeared within 3 weeks after surgery in all patients. ${ }^{34}$ Wound healing delay occurred in 3 cases but did not require additional surgery. Therefore, the incidence of 30-day morbidity was $2.1 \%$ (3/141).

During follow-up periods, none of the patients developed ischemic stroke, but 1 patient (1.1\%) experienced hemorrhagic stroke. At initial presentation, this 49-yearold male patient developed intracerebral hemorrhage in the left temporal lobe and a diagnosis of unilateral moyamoya disease was made. The patient underwent STAMCA double anastomosis and EDMAPS on the left side. His clinical course was good, but he suffered rebleeding in the left putamen associated with intraventricular hema- 
TABLE 1. Summary of 93 patients who were followed up for at least 5 years after STA-MCA anastomosis and EDMAPS for moyamoya disease

\begin{tabular}{|c|c|c|c|}
\hline & $\begin{array}{c}\text { Pediatric } \\
\text { Group }\end{array}$ & $\begin{array}{l}\text { Adult } \\
\text { Group }\end{array}$ & Total \\
\hline No. of patients & 35 & 58 & 93 \\
\hline No. of treated hemispheres & 54 & 87 & 141 \\
\hline Age, yrs & $8.8 \pm 3.8$ & $42.3 \pm 12.0$ & $30.0 \pm 19.0$ \\
\hline \multicolumn{4}{|l|}{ Sex } \\
\hline Male & 15 & 14 & 29 \\
\hline Female & 20 & 44 & 64 \\
\hline \multicolumn{4}{|l|}{ Initial diagnosis } \\
\hline TIA & 7 & 35 & 42 \\
\hline Ischemic stroke & 28 & 10 & 38 \\
\hline Hemorrhagic stroke & 0 & 10 & 10 \\
\hline Asymptomatic & 0 & 3 & 3 \\
\hline \multicolumn{4}{|l|}{ Comorbidity } \\
\hline Hypertension & $0(0 \%)$ & $12(20.7 \%)$ & $12(12.9 \%)$ \\
\hline Hyperlipidemia & $0(0 \%)$ & $8(13.8 \%)$ & $8(8.6 \%)$ \\
\hline Diabetes & $0(0 \%)$ & $3(5.2 \%)$ & $3(3.2 \%)$ \\
\hline Smoking & $0(0 \%)$ & $7(12.1 \%)$ & $7(7.5 \%)$ \\
\hline Baseline mRS score & $0.31 \pm 0.76$ & $0.31 \pm 0.73$ & $0.31 \pm 0.74$ \\
\hline \multicolumn{4}{|l|}{ Suzuki stage } \\
\hline 3 & 15 & 18 & 33 \\
\hline 4 & 12 & 23 & 35 \\
\hline 5 & 7 & 12 & 19 \\
\hline 6 & 1 & 5 & 6 \\
\hline Preop disease period, mos & $4.2 \pm 2.6$ & $8.0 \pm 7.2$ & $6.3 \pm 4.0$ \\
\hline Follow-up, yrs & $10.9 \pm 4.8$ & $10.2 \pm 4.3$ & $10.5 \pm 4.4$ \\
\hline
\end{tabular}

Values are presented as number of patients or mean \pm SD unless otherwise indicated.

toma 9.5 years after surgery. Therefore, the risk of any stroke after STA-MCA anastomosis and EDMAPS was $0.10 \%$ per patient-year. At the last follow-up, modified Rankin Scale (mRS) scores were graded as 0 in 80 patients, 1 in 8 patients, 2 in 2, 3 in 2, and 4 in 1 patient. The mean $\mathrm{mRS}$ for all 93 patients changed from $0.32 \pm 0.74$ at initial presentation to $0.24 \pm 0.70$ at the last follow-up. There was no significant change between the two periods.

\section{Radiological Outcome}

Repeated MRA revealed disease progression in 19 hemispheres (13.5\%) of 15 patients (16.1\%) during a mean of 10.5 years (Table 2). Therefore, the annual risk of disease progression was $1.5 \%$ per patient-year. Female predominance was observed (11/15). The clinical diagnosis at initial presentation was TIA or ischemic stroke in 14 of the 15 patients, with 1 patient asymptomatic.

Disease progression was observed in the contralateral ICA in 9 patients who underwent STA-MCA anastomosis and EDMAPS on the unilateral hemisphere at initial presentation. Of these, 7 patients were younger than 20 years. Disease progression in the contralateral ICA occurred within 5 years after initial surgery in 6 patients and caused TIA in 7 of 9 patients. STA-MCA anastomosis and EDMAPS were performed on the contralateral hemisphere in 7 of 9 patients. All of these patients remained free from any cerebrovascular events at the time of this report.

As shown in Table 2, disease progression in the PCA was observed in 11 hemispheres of 8 patients. Their age varied widely, from 1 to 52 years. The timing of disease progression after initial surgery also widely differed among these patients, varying from 0.5 to 15 years (mean $5.4 \pm 4.4$ years). Disease progression occurred in the bilateral PCAs of $3 / 8$ patients or in both the contralateral ICA and unilateral PCA in 2/8 patients. Transient neurological symptoms such as homonymous hemianopsia were recorded in 6 hemispheres of 8 patients. Of these, indirect bypass (encephalo-duro-pericranio-synangiosis [EDPS]) was performed in 4 hemispheres of 4 patients because the acceptable recipient artery could not be found in the operative field. After additional surgery, all 4 patients experienced no cerebrovascular events.

\section{Discussion \\ Late Clinical Outcomes}

According to previous reports by us and other investigators, surgical revascularization has substantially improved short-term to midterm ( $<5$ years) outcomes in patients with moyamoya disease. However, a period of 5 or 6 years is too short to fully assess the benefits of bypass surgery for moyamoya disease, because most of the patients are children or young adults and have a much longer lifetime after surgery than before. As the results of the present study clearly indicate, treatment with STA-MCA anastomosis and EDMAPS may lead to satisfactory outcomes in both pediatric and adult patients with moyamoya disease. During the follow-up period of the current study, which ranged from 5 to 20 years (mean 10.5 years), 92 of 93 patients $(98.9 \%)$ were free from any stroke and death, although 1 patient experienced rebleeding in the ipsilateral hemisphere 9.5 years postsurgery $(0.10 \%$ per patient-year).

Recently, two Japanese groups have published their long-term ( $>10$ years) outcome results in a large cohort of surgically treated children with moyamoya disease. Namely, Mukawa et al. (2012) surveyed 14.3-year outcomes after indirect bypass (encephalo-duro-arteriosynangiosis [EDAS]) in 172 pediatric patients. In about $80 \%$ of patients the results were graded as good functional outcome (mRS score 0-2). Of the 172 patients, however, 6 developed ischemic $(n=3)$ or hemorrhagic $(n=9)$ stroke at 8-22 years after EDAS. Based on these results, the risk of stroke in this large cohort at 10,20 , and 30 years postEDAS was $0.8 \%, 6.3 \%$, and $10.0 \%$, respectively. ${ }^{31}$ Funaki et al. (2014) also evaluated the 18.1-year outcome after STA-MCA anastomosis and EMS in 56 pediatric patients and found that 4 of 56 patients developed ischemic ( $\mathrm{n}=$ 1) or hemorrhagic $(n=4)$ stroke at 2-20 years postsurgery. The 10-, 20-, and 30-year cumulative risks of stroke were $1.8 \%, 7.3 \%$, and $13.1 \%$, respectively. ${ }^{4}$ Therefore, STA-MCA anastomosis and EDMAPS may lead to better long-term ( $>5$ years) outcomes for pediatric patients than EDAS or STA-MCA anastomosis combined with EMS. 


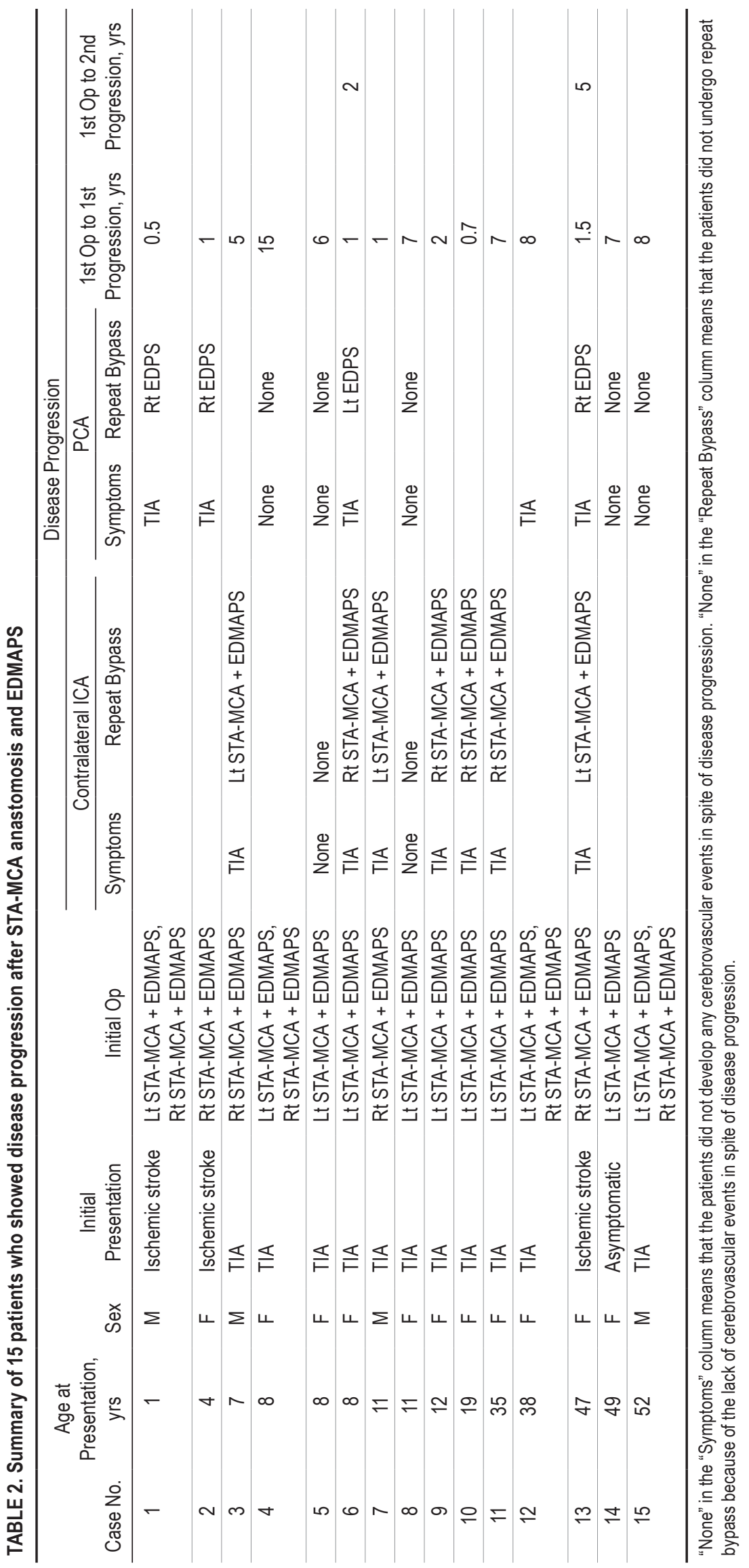


However, further study is essential to follow up these pediatric patients for a longer time period extending to adulthood.

On the other hand, there are almost no reports of study results for long-term ( $>10$ years) outcomes in adult moyamoya patients who underwent bypass surgery, although many reports of clinical results during 5- to 6-year followup periods have been published. Only Bao et al. (2018) performed EDAS for 145 adult patients and followed them up for approximately 12 years. These authors reported $1-, 5-$, and 10-year estimated cumulative stroke rates of $2.1 \%, 6.8 \%$, and $8.9 \%$, respectively. Bao et al. concluded that bilateral involvement was a common significant factor in any strokes and that hypertension was a risk factor for ischemic strokes during follow-up. ${ }^{1}$ The values reported by Bao et al. were somewhat larger than those of the present study. Therefore, the present study is to our knowledge the first to clearly show that STA-MCA anastomosis and EDMAPS may also lead to the best long-term ( $>5$ years) outcomes reported to date for adult patients with moyamoya disease.

\section{Late Radiological Outcome and Repeat Bypass}

Previously, we analyzed the findings on serial MRA in adult patients and reported that disease progression occurred in 15 of 86 nonoperated hemispheres (17.4\%), or in 15 of 63 patients $(23.8 \%)$, during the first 6 years after bypass surgery. ${ }^{24}$ The present study clearly shows that moyamoya patients are exposed to the risk for disease progression even thereafter, and thus it should be recognized that disease progression is likely to occur during at least the first 15 years after surgery.

In the present study, disease progression occurred in the contralateral ICA territory in 9 patients during the followup period. Most of them (7/9) were younger than 20 years and showed disease progression within 5 years after the initial surgery. These findings are supported by previous reports. Namely, Yeon et al. (2011) reported that contralateral progression occurred in 8 of 45 children (17.8\%) with unilateral moyamoya disease within 3 years of initial diagnosis. All of these patients were younger than 9 years. ${ }^{39}$ Park et al. (2011) also reported that 20 of 34 pediatric patients $(58.8 \%)$ with unilateral moyamoya disease experienced contralateral progression within approximately 3 years. ${ }^{33}$

In our study, disease progression in the PCA occurred in 11 hemispheres of 8 patients. The features of PCA progression largely differed from those of contralateral progression. The age of the study patients varied from 1 to 52 years at initial presentation, and there was very wide variation in the timing of disease progression, from 0.5 to 15 years after initial surgery. About half (6/11) of the PCA lesions caused TIA. There are few reports regarding disease progression in the PCA in surgically treated moyamoya patients during long-term follow-up periods. However, it should be noted that occlusive arterial lesions progressed in both carotid and PCA territories. ${ }^{24}$ Huang et al. (2009) reported that occlusive lesions in the PCA progressed on $18 / 38$ sides (47.4\%) after surgical revascularization for anterior circulation..$^{12}$ Lee et al. (2013) found that 29/166 sides (17\%) showed PCA disease progression in pediatric moya- moya disease patients during a mean follow-up period of 8.3 years. The mean timing of disease progression after the initial surgery was 4.9 years, with the latest onset at 10.8 years. ${ }^{26}$ Based on these observations, we should recognize that disease progression in the PCA is not as rare as was previously considered. Furthermore, occlusive lesions in the PCA may be key factors leading to hemorrhagic stroke in adult moyamoya disease. ${ }^{3}$ Therefore, we should be aware that disease progression in the PCA may occur in a delayed fashion, and we should follow up moyamoya patients for longer than 10 years after bypass surgery in order not to miss disease progression in the PCA, which carries the potential risk for TIA or ischemic or hemorrhagic stroke.

In this study, repeat bypass surgery was indicated when ischemic symptoms were severe due to dense cerebral ischemia in the territory of the contralateral ICA $(n=7)$ or PCA $(n=4)$. For the former, STA-MCA anastomosis combined with EDMAPS was performed. For the latter, however, only an indirect bypass procedure (EDPS) was performed because of the lack of an acceptable recipient artery. Recently, several reported studies have demonstrated the feasibility and beneficial effects of repeat bypass for both the anterior and posterior circulation. ${ }^{8,17,25,36,37}$ Hayashi et al. (2009) reported 3 patients with moyamoya disease who underwent occipital artery (OA) to PCA anastomosis and indirect bypass because of disease progression in the PCA.? Lee et al. (2014) also performed indirect bypass onto the PCA territory for 41 children with delayed PCA insufficiency, and these authors observed that this treatment yielded good clinical outcomes. ${ }^{25}$ We also performed OA-MCA $(n=3)$ or OA-PCA anastomosis $(n=8)$ combined with indirect bypass in patients who showed disease progression in the PCA after surgical revascularization for the anterior circulation. ${ }^{17}$ Furthermore, we very recently reported that repeat bypass is feasible and very effective for both the carotid and PCA territories on the basis of our experience in 20 patients with refractory moyamoya disease. ${ }^{37}$

\section{Conclusions}

STA-MCA anastomosis and ultimate indirect bypass (EDMAPS) may be one of the best choices of surgical treatment to prevent further cerebrovascular events for longer than 10 years in moyamoya patients by widely providing surgical collaterals to both the MCA and ACA territories. However, regular follow-up should be planned for longer than 10 years postsurgery to identify disease progression in the territory of the contralateral carotid artery and PCA and prevent late cerebrovascular events. Repeat bypass surgery is feasible and effective to resolve ischemic attacks in both carotid and PCA territories.

\section{Acknowledgments}

This study was partly supported by a grant from the Research Committee on Moyamoya Disease, sponsored by the Ministry of Health, Labor and Welfare of Japan (grant no. H29-032).

\section{References}

1. Bao XY, Zhang Y, Wang QN, Zhang Q, Wang H, Zhang ZS, 
et al: Long-term outcomes after encephaloduroarteriosynangiosis in adult patients with moyamoya disease presenting with ischemia. World Neurosurg 115:e482-e489, 2018

2. Duan L, Bao XY, Yang WZ, Shi WC, Li DS, Zhang ZS, et al: Moyamoya disease in China: its clinical features and outcomes. Stroke 43:56-60, 2012

3. Funaki T, Takahashi JC, Houkin K, Kuroda S, Takeuchi S, Fujimura M, et al: Angiographic features of hemorrhagic moyamoya disease with high recurrence risk: a supplementary analysis of the Japan Adult Moyamoya Trial. J Neurosurg 128:777-784, 2018

4. Funaki T, Takahashi JC, Takagi Y, Yoshida K, Araki Y, Kikuchi T, et al: Incidence of late cerebrovascular events after direct bypass among children with moyamoya disease: a descriptive longitudinal study at a single center. Acta Neurochir (Wien) 156:551-559, 2014

5. Guzman R, Lee M, Achrol A, Bell-Stephens T, Kelly M, Do $\mathrm{HM}$, et al: Clinical outcome after 450 revascularization procedures for moyamoya disease. Clinical article. J Neurosurg 111:927-935, 2009

6. Hallemeier CL, Rich KM, Grubb RL Jr, Chicoine MR, Moran CJ, Cross DT III, et al: Clinical features and outcome in North American adults with moyamoya phenomenon. Stroke 37:1490-1496, 2006

7. Hayashi T, Shirane R, Tominaga T: Additional surgery for postoperative ischemic symptoms in patients with moyamoya disease: the effectiveness of occipital artery-posterior cerebral artery bypass with an indirect procedure: technical case report. Neurosurgery 64:E195-E196, 2009

8. Hori S, Kashiwazaki D, Akioka N, Hamada H, Kuwayama N, Kuroda S: [Repeat bypass surgery for intracranial hemorrhage 30 years after indirect bypass for moyamoya disease.] No Shinkei Geka 42:347-353, 2014 (Japanese)

9. Hori S, Kashiwazaki D, Akioka N, Hayashi T, Hori E, Umemura K, et al: Surgical anatomy and preservation of the middle meningeal artery during bypass surgery for moyamoya disease. Acta Neurochir (Wien) 157:29-36, 2015

10. Houkin K, Kamiyama H, Abe H, Takahashi A, Kuroda S: Surgical therapy for adult moyamoya disease. Can surgical revascularization prevent the recurrence of intracerebral hemorrhage? Stroke 27:1342-1346, 1996

11. Houkin K, Kuroda S, Nakayama N: Cerebral revascularization for moyamoya disease in children. Neurosurg Clin $\mathbf{N}$ Am 12:575-584, ix, 2001

12. Huang AP, Liu HM, Lai DM, Yang CC, Tsai YH, Wang KC, et al: Clinical significance of posterior circulation changes after revascularization in patients with moyamoya disease. Cerebrovasc Dis 28:247-257, 2009

13. Ishikawa T, Houkin K, Kamiyama H, Abe H: Effects of surgical revascularization on outcome of patients with pediatric moyamoya disease. Stroke 28:1170-1173, 1997

14. Ishikawa T, Kamiyama H, Kuroda S, Yasuda H, Nakayama N, Takizawa K: Simultaneous superficial temporal artery to middle cerebral or anterior cerebral artery bypass with pansynangiosis for Moyamoya disease covering both anterior and middle cerebral artery territories. Neurol Med Chir (Tokyo) 46:462-468, 2006

15. Kamiyama H, Takahashi A, Houkin K, Mabuchi S, Abe H: Visualization of the ostium of an arteriotomy in bypass surgery. Neurosurgery 33:1109-1110, 1993

16. Karasawa J, Touho H, Ohnishi H, Miyamoto S, Kikuchi H: Long-term follow-up study after extracranial-intracranial bypass surgery for anterior circulation ischemia in childhood moyamoya disease. J Neurosurg 77:84-89, 1992

17. Kazumata K, Kamiyama H, Saito H, Maruichi K, Ito M, Uchino H, et al: Direct anastomosis using occipital artery for additional revascularization in moyamoya disease after combined superficial temporal artery-middle cerebral artery and indirect bypass. Oper Neurosurg (Hagerstown) 13:213-223, 2017
18. Kuroda S, Hashimoto N, Yoshimoto T, Iwasaki Y: Radiological findings, clinical course, and outcome in asymptomatic moyamoya disease: results of multicenter survey in Japan. Stroke 38:1430-1435, 2007

19. Kuroda S, Houkin K: Bypass surgery for moyamoya disease: concept and essence of surgical techniques. Neurol Med Chir (Tokyo) 52:287-294, 2012

20. Kuroda S, Houkin K: Moyamoya disease: current concepts and future perspectives. Lancet Neurol 7:1056-1066, 2008

21. Kuroda S, Houkin K, Ishikawa T, Nakayama N, Ikeda J, Ishii $\mathrm{N}$, et al: Determinants of intellectual outcome after surgical revascularization in pediatric moyamoya disease: a multivariate analysis. Childs Nerv Syst 20:302-308, 2004

22. Kuroda S, Houkin K, Ishikawa T, Nakayama N, Iwasaki Y: Novel bypass surgery for moyamoya disease using pericranial flap: its impacts on cerebral hemodynamics and long-term outcome. Neurosurgery 66:1093-1101, 2010

23. Kuroda S, Houkin K, Kamiyama H, Mitsumori K, Iwasaki Y, Abe H: Long-term prognosis of medically treated patients with internal carotid or middle cerebral artery occlusion: can acetazolamide test predict it? Stroke 32:2110-2116, 2001

24. Kuroda S, Ishikawa T, Houkin K, Nanba R, Hokari M, Iwasaki Y: Incidence and clinical features of disease progression in adult moyamoya disease. Stroke 36:2148-2153, 2005

25. Lee JY, Choi YH, Cheon JE, Paeng JC, Ryu HW, Kim KJ, et al: Delayed posterior circulation insufficiency in pediatric moyamoya disease. J Neurol 261:2305-2313, 2014

26. Lee JY, Kim SK, Cheon JE, Choi JW, Phi JH, Kim IO, et al: Posterior cerebral artery involvement in moyamoya disease: initial infarction and angle between PCA and basilar artery. Childs Nerv Syst 29:2263-2269, 2013

27. Lee SB, Kim DS, Huh PW, Yoo DS, Lee TG, Cho KS: Longterm follow-up results in 142 adult patients with moyamoya disease according to management modality. Acta Neurochir (Wien) 154:1179-1187, 2012

28. Matsushima T, Inoue K, Kawashima M, Inoue T: History of the development of surgical treatments for moyamoya disease. Neurol Med Chir (Tokyo) 52:278-286, 2012

29. Miyamoto S, Akiyama Y, Nagata I, Karasawa J, Nozaki K, Hashimoto N, et al: Long-term outcome after STA-MCA anastomosis for moyamoya disease. Neurosurg Focus 5(5):e5, 1998

30. Miyamoto S, Yoshimoto T, Hashimoto N, Okada Y, Tsuji I, Tominaga $T$, et al: Effects of extracranial-intracranial bypass for patients with hemorrhagic moyamoya disease: results of the Japan Adult Moyamoya Trial. Stroke 45:1415-1421, 2014

31. Mukawa M, Nariai T, Matsushima Y, Tanaka Y, Inaji M, Maehara T, et al: Long-term follow-up of surgically treated juvenile patients with moyamoya disease. J Neurosurg Pediatr 10:451-456, 2012

32. Okada Y, Shima T, Nishida M, Yamane K, Yamada T, Yamanaka C: Effectiveness of superficial temporal artery-middle cerebral artery anastomosis in adult moyamoya disease: cerebral hemodynamics and clinical course in ischemic and hemorrhagic varieties. Stroke 29:625-630, 1998

33. Park EK, Lee YH, Shim KW, Choi JU, Kim DS: Natural history and progression factors of unilateral moyamoya disease in pediatric patients. Childs Nerv Syst 27:1281-1287, 2011

34. Sasamori T, Kuroda S, Nakayama N, Iwasaki Y: Incidence and pathogenesis of transient cheiro-oral syndrome after surgical revascularization for moyamoya disease. Neurosurgery 67:1054-1060, 2010

35. Suzuki J, Takaku A: Cerebrovascular "moyamoya" disease. Disease showing abnormal net-like vessels in base of brain. Arch Neurol 20:288-299, 1969

36. Teo M, Johnson J, Steinberg GK: Strategies for and outcome of repeat revascularization surgery for moyamoya disease: an American institutional series. Neurosurgery 81:852-859, 2017 
37. Uchino H, Kashiwazaki D, Akioka N, Koh M, Kuwayama N, Houkin K, et al: Strategy and effect of repeat bypass surgery for anterior/posterior circulation in refractory moyamoya disease. J Neurosurg [epub ahead of print May 31, 2019; DOI: 10.3171/2019.3.JNS181979]

38. Uchino H, Kuroda S, Hirata K, Shiga T, Houkin K, Tamaki N: Predictors and clinical features of postoperative hyperperfusion after surgical revascularization for moyamoya disease: a serial single photon emission CT/positron emission tomography study. Stroke 43:2610-2616, 2012

39. Yeon JY, Shin HJ, Kong DS, Seol HJ, Kim JS, Hong SC, et al: The prediction of contralateral progression in children and adolescents with unilateral moyamoya disease. Stroke 42:2973-2976, 2011

\section{Disclosures}

The authors report no conflict of interest concerning the materials or methods used in this study or the findings specified in this paper.

\section{Author Contributions}

Conception and design: Kuroda. Acquisition of data: Kuroda, Nakayama, Yamamoto, Kashiwazaki, Uchino, Saito, Hori, Akioka, Kuwayama. Analysis and interpretation of data: Kuroda. Drafting the article: Kuroda. Critically revising the article: Kuroda. Reviewed submitted version of manuscript: Kuroda. Approved the final version of the manuscript on behalf of all authors: Kuroda. Study supervision: Houkin.

\section{Correspondence}

Satoshi Kuroda: Graduate School of Medicine and Pharmaceutical Science, University of Toyama, Toyama, Japan. skuroda@ med.u-toyama.ac.jp. 